\title{
Clinical Study \\ Influence of Helicobacter pylori Infection on the Small Intestinal Mucosa
}

\author{
Mitsunori Maeda, Masakazu Nakano, and Hideyuki Hiraishi \\ Department of Gastroenterology, Dokkyo Medical University, Tochigi, Mibu 321-0293, Japan \\ Correspondence should be addressed to Mitsunori Maeda; maedas42@dokkyomed.ac.jp
}

Received 4 June 2013; Accepted 19 August 2013

Academic Editors: B. Braden, P. Figueiredo, and C. Romano

Copyright (C) 2013 Mitsunori Maeda et al. This is an open access article distributed under the Creative Commons Attribution License, which permits unrestricted use, distribution, and reproduction in any medium, provided the original work is properly cited.

\begin{abstract}
Background/Aims. To investigate the role of Helicobacter pylori infection in the development of enteritis (small intestinal mucosal injury). Methodology. Between April 2007 and January 2013, 99 patients undergoing capsule endoscopy (CE) were tested for antiH. pylori immunoglobulin $\mathrm{G}$ antibody ( $\mathrm{Hp}$-IgG) using an enzyme-linked immunosorbent assay (ELISA). None of the patients had been treated for $\mathrm{H}$. pylori infection or diagnosed as having Crohn's disease or any other clinically apparent small intestinal disorders prior to the CE. Results. The overall $\mathrm{Hp}$-IgG-positive rate was $26.3 \%$. The incidence of enteritis, as diagnosed by CE, tended to be lower in the $H p$-IgG-positive patients (23.1\%) than in the $H p$-IgG-negative patients $(38.4 \%)(P=0.140)$. When patients receiving aspirin or nonsteroidal anti-inflammatory drugs (NSAIDs), well-known causes of enteritis, were excluded, the incidence of enteritis in the $\mathrm{Hp}$-IgG-positive patients $(11.7 \%)$ was significantly lower than that in the $\mathrm{Hp}$-IgG-negative patients $(43.7 \%)(P=0.005)$. A binomial logistic regression analysis revealed a significant negative relationship between $H p$-IgG positivity and the presence of enteritis in patients receiving neither aspirin nor NSAIDs $(P=0.017)$. Conclusions. Our data indicated that $H$. pylori positivity was inversely associated with the prevalence of enteritis.
\end{abstract}

\section{Introduction}

Helicobacter pylori is a Gram-negative spiral-shaped microaerophilic bacterium that is known to colonize the gastric mucosa in humans [1]. Although $H$. pylori infection has been reported to be associated with an increased risk of gastric ulcer, duodenal ulcer, and atrophic gastritis [2,3], little information is available on the possible association of $H$. pylori infection with enteritis (small intestinal mucosal injury).

The aim of the present study was to assess the incidence of enteritis by capsule endoscopy (CE) in anti-H. pylori immunoglobulin G antibody ( $\mathrm{Hp}$-IgG)-positive and $\mathrm{Hp}$-IgGnegative patients.

\section{Methodology}

2.1. Patients. Between April 2007 and January 2013, НpIgG antibody was prospectively measured in 99 patients (70 men, 29 women; mean age, $63.4 \pm 16.8$ years; age range, 22-86 years) with obscure gastrointestinal bleeding (OGIB) at the Department of Gastroenterology, Dokkyo Medical
University. Prior to July 2012, OGIB was the only indication for $\mathrm{CE}$ that was covered by the national health insurance system of Japan.

Patients diagnosed as having Crohn's disease, small bowel tumor, suspected ileus or gastrointestinal stricture, or radiation enterocolitis or having a history of intra-abdominal surgery or pacemaker implantation were excluded from this study. In all patients, the CE, the upper and lower endoscopy, was performed within 60 days of the last bleeding episode. The characteristics of the 99 patients are summarized in Table 1.

This study was conducted with the approval of the ethics committee of Dokkyo Medical University, and written informed consent was obtained from all the patients.

2.2. Capsule Endoscopy. CE for identifying small intestinal lesions was performed using an M2A/PillCamTM SB or SB2 (Given Imaging Ltd., Yokneam, Israel). After the patients had fasted for 8 hours, they received $4 \mathrm{~mL}$ ( $40 \mathrm{mg}$ ) of simethicone just before swallowing the capsule endoscope. The patients were allowed to drink water from 2 hours and eat from 4 
TABLE 1: Patient characteristics $(n=99)$.

\begin{tabular}{|c|c|c|c|}
\hline & $H p(+)$ & $H p(-)$ & $P$ value \\
\hline Sex (male/female) & $18: 9$ & $52: 21$ & \\
\hline Age (years) & $68.0 \pm 12.0$ & $61.7 \pm 18.0$ & 0.103 \\
\hline Time from last bleeding episode until CE (days) & $12.6 \pm 13.4$ & $12.3 \pm 16.3$ & 0.938 \\
\hline \multicolumn{4}{|l|}{ Past and present illness } \\
\hline History of gastroduodenal ulcer & $23.10 \%$ & $10.90 \%$ & 0.13 \\
\hline Gastritis & $84.60 \%$ & $72.60 \%$ & 0.224 \\
\hline Neurological disorder (cerebral infarction, etc.) & $26.90 \%$ & $13.70 \%$ & 0.127 \\
\hline Heart disease (valvular disease, etc.) & $23.10 \%$ & $42.50 \%$ & 0.081 \\
\hline Liver disease (chronic hepatitis, etc.) & $3.80 \%$ & $15.10 \%$ & 0.135 \\
\hline Diabetes mellitus & $15.40 \%$ & $26.00 \%$ & 0.274 \\
\hline
\end{tabular}

CE: capsule endoscopy.

hours after swallowing the capsule. The recorder was removed after 8 hours, and images were analyzed using the RAPID (Reporting and Processing of Images and Data, 2-6.5; Given Imaging Ltd.) software.

Enteritis was defined as small intestinal mucosal injury, namely, the presence of ulceration, erosions, or erythema in the mucosa of the small intestine, as detected by CE.

2.3. Helicobacter pylori Infection Status. The H. pylori infection status was examined by enzyme-linked immunosorbent assay (ELISA) to detect $H p$-IgG. The reported $H p$-IgG-positive rate among patients with $H$. pylori infection is in the range of $92.0 \%$ and $97.1 \%[4,5]$.

2.4. Statistical Analysis. Differences in the patient characteristics were tested for statistical significance by the student's paired $t$-test or Welch's $t$-test. A binomial logistic regression analysis was conducted to analyze the relationship between enteritis as the dependent variable and a history of gastroduodenal ulcer, gastrointestinal cancer, neurological disorder, heart disease, liver disease, serum C-reactive protein (CRP), $\mathrm{Hp}$-IgG positivity, and aspirin/nonsteroidal anti-inflammatory drugs (NSAIDs) used as independent variables. The partial regression coefficient, odds ratios (OR), and 95\% confidence intervals $(\mathrm{CI})$ were calculated, and $P$ values $\leq 0.05$ were considered to indicate statistical significance. The statistical analyses were performed using the IBM SPSS 19.0 software for windows (IBM Corporation, Armonk, NY, USA).

\section{Results}

3.1. Relationship between $H$. pylori Infection and Enteritis. Of the 99 patients undergoing CE for OGIB, 26 were found to be Hp-IgG-positive (26.3\%).

The incidence of enteritis among the $26 \mathrm{Hp}$-IgG-positive patients $(6 / 26 ; 23.1 \%)$ tended to be lower than that in the remaining $73 \mathrm{Hp}$-IgG-negative patients $(28 / 73 ; 38.4 \%)(P=$ 0.140; Figure 1).

3.2. Incidence of Enteritis among Patients Not Receiving Aspirin/NSAIDs. Because aspirin/NSAID use is well known to be associated with enteritis, the incidence of enteritis was

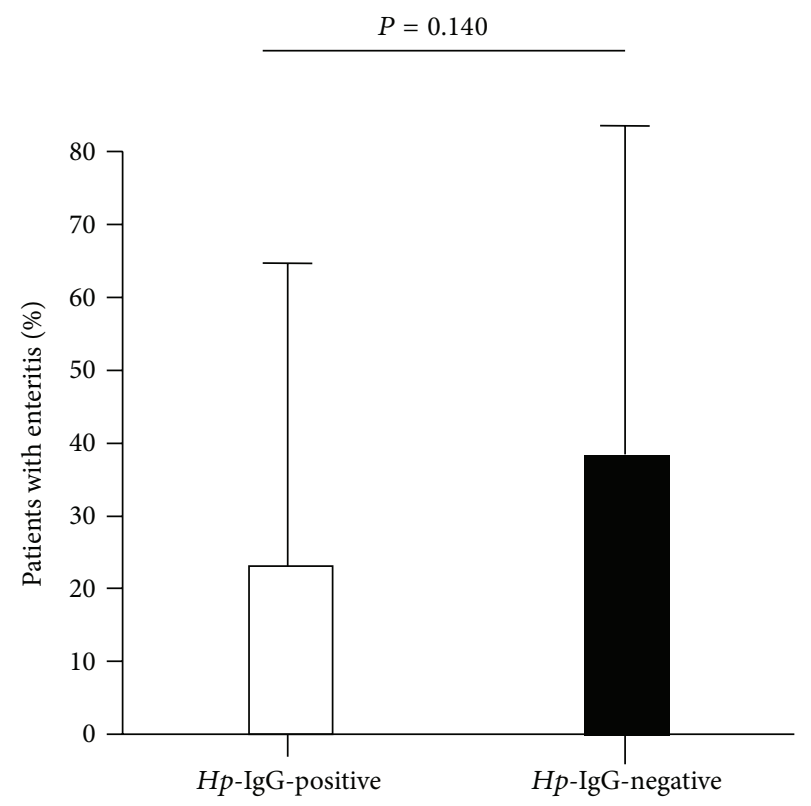

FIgure 1: Incidence of enteritis in $\mathrm{Hp}$-IgG-positive and $\mathrm{Hp}$-IgGnegative patients.

determined in 65 of the 99 patients who were not receiving aspirin/NSAIDs. Analysis revealed that among the patients not receiving aspirin/NSAIDs, the incidence of enteritis in the $17 \mathrm{Hp}$-IgG-positive patients $(2 / 17 ; 11.8 \%)$ was significantly lower than that in the $48 \mathrm{Hp}$-IgG-negative patients $(21 / 48$; 43.7\%) $(P=0.005$; Figure 2).

\subsection{Incidence of Enteritis in Hp-IgG-Positive Patients Receiving} Aspirin and/or NSAIDs with or without a Proton Pump Inhibitor. Because it has been reported that proton pump inhibitors (PPIs) can exacerbate NSAID-induced small intestinal mucosal injury by inducing dysbiosis [6] and also exert both a bactericidal and a bacteriostatic effect against $H$. pylori [79], we compared the incidence of enteritis between a patient group receiving a PPI and a patient group not receiving PPI therapy among $26 \mathrm{Hp}$-IgG-positive patients receiving aspirin/ NSAIDs. The results revealed that the incidence of enteritis 


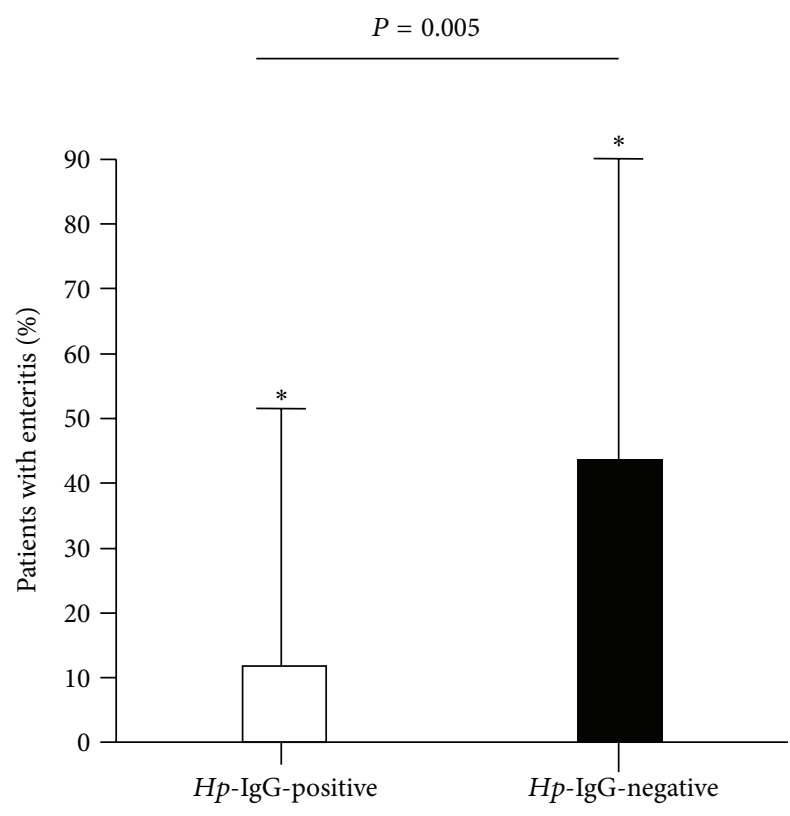

Figure 2: Incidence of enteritis in $\mathrm{Hp}$-IgG-positive and $\mathrm{Hp}$-IgGnegative patients receiving neither aspirin nor NSAIDs.

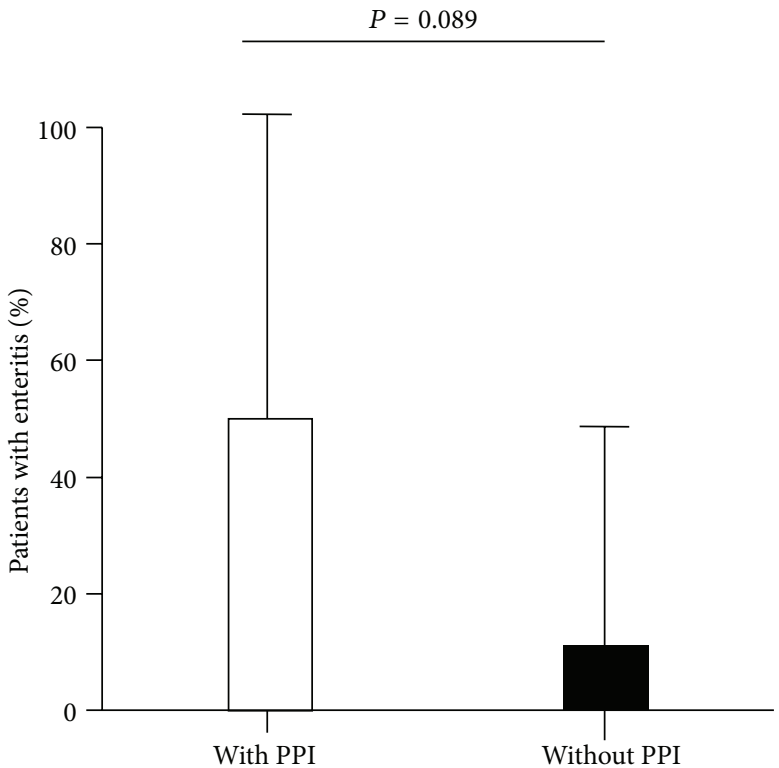

FIGURE 3: Incidence of enteritis in Hp-IgG-positive patients receiving aspirin/NSAIDs with or without a PPI.

tended to be higher in the patient group receiving PPI therapy $(4 / 8 ; 50.0 \%)$ than in the patient group not receiving PPI therapy $(2 / 18 ; 11.1 \%)(P=0.089$; Figure 3$)$.

3.4. Incidence of Enteritis in Hp-IgG-Positive Patients Not on Aspirin/NSAIDs, Receiving or Not Receiving PPI Therapy. To determine the effect of only PPIs on the small intestinal mucosa, we compared the incidence of enteritis between a patient group receiving PPI therapy and a patient group not receiving PPI therapy among $17 \mathrm{Hp}$-IgG-positive patients not

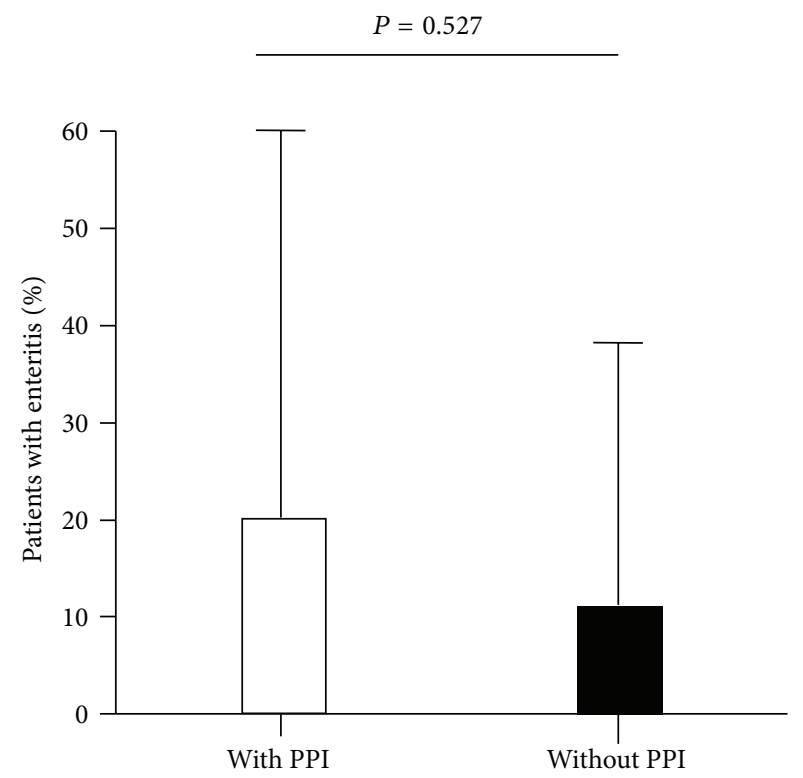

FIGURE 4: Incidence of enteritis in $\mathrm{Hp}$-IgG-positive patients not on aspirin/NSAIDs, receiving or not receiving PPI therapy.

receiving aspirin/NSAIDs. The results revealed enteritis in 1 of $5(20.0 \%)$ patients receiving PPI therapy, as compared to 1 of $12(8.3 \%)$ patients not receiving PPI therapy $(P=0.526$; Figure 4).

3.5. Incidence of Enteritis in Hp-IgG-Negative Patients Receiving Aspirin/NSAIDs with or without PPI. To examine whether PPI therapy may have the same effect in $\mathrm{Hp}$-IgG-negative patients as in $H p$-IgG-positive patients, we compared the incidence of enteritis between a group receiving PPI therapy and a group not receiving PPI therapy among $25 \mathrm{Hp}$ IgG-negative patients receiving aspirin/NSAIDs. The results revealed enteritis in 3 of 8 (37.5\%) patients receiving PPI therapy, as compared to 4 of 17 (23.5\%) patients not receiving PPI therapy $(P=0.489$; Figure 5$)$.

3.6. Factors Associated with Enteritis. A binomial logistic regression analysis for patients not receiving aspirin/NSAIDs revealed that enteritis positively correlated with the male sex, advanced age, neurological disorder, elevated serum CRP, and history of gastroduodenal ulcer and negatively correlated with $\mathrm{Hp}$-IgG-positivity, heart disease, liver disease, diabetes mellitus, and gastritis (Table 2). Only the negative relationship with $H p$-IgG positivity was statistically significant at $P<$ 0.05 .

\section{Discussion}

H. pylori infection is known to be associated with many gastrointestinal disorders such as gastritis, gastric ulcer, gastroesophageal reflux disease (GERD), gastric cancer, and NSAID/ aspirin-induced gastric injury $[2,3,10,11]$. Although $H$. pylori presence has also been detected in the small intestine [12], there are few reports on how $H$. pylori may affect the small intestinal mucosa. Until now, only small intestinal bacterial 
TABLE 2: Factors associated with enteritis (binomial logistic regression analysis).

\begin{tabular}{|c|c|c|c|c|}
\hline Factor & Partial regression coefficient & Odds ratio & 95\% confidence interval & $P$ value \\
\hline Sex & 0.316 & 1.371 & $0.309-6.078$ & 0.678 \\
\hline Age & 0.023 & 1.023 & $0.980-1.068$ & 0.297 \\
\hline Presence of heart disease & -0.21 & 0.81 & $0.183-3.582$ & 0.781 \\
\hline Presence of liver disease & -0.869 & 0.42 & $0.035-5.027$ & 0.493 \\
\hline Presence of neurological disorder & 2.137 & 8.477 & $0.692-103.820$ & 0.094 \\
\hline Presence of diabetes mellitus & -0.485 & 0.616 & $0.109-3.468$ & 0.582 \\
\hline CRP value & 0.096 & 1.101 & $0.331-3.659$ & 0.876 \\
\hline$H p$-IgG-positive & -2.976 & 0.051 & $0.004-0.590$ & $0.017^{*}$ \\
\hline Presence of gastritis & -0.071 & 0.931 & $0.220-3.945$ & 0.923 \\
\hline History of gastroduodenal ulcer & 0.222 & 1.248 & $0.201-7.762$ & 0.812 \\
\hline
\end{tabular}

Hp: helicobacter pylori; CRP: C-reactive protein; NSAIDs: nonsteroidal anti-inflammatory drugs. ${ }^{*}$ A statistically significant negative correlation was shown between Hp-IgG-positive and enteritis in Table 2.

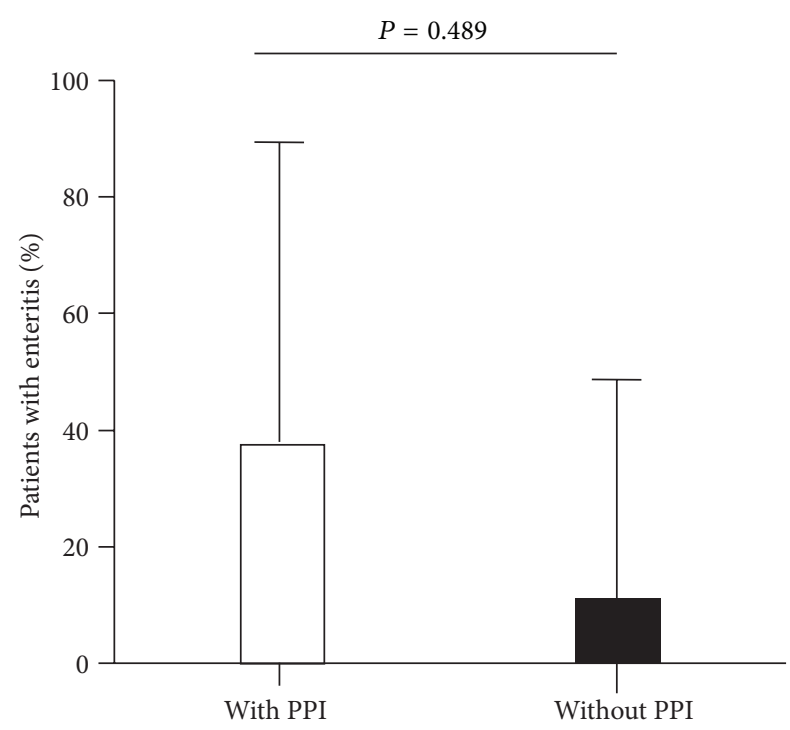

FIGURE 5: Incidence of enteritis in $H p$-IgG-negative patients receiving aspirin/NSAIDs with or without a PPI.

overgrowth (SIBO) [13-16] and the phenotype of Crohn's disease [17] have been reported to be associated with $H$. pylori in the small intestine.

Our results indicated that the incidence of small intestinal mucosal injury tended to be lower in $H p$-infected patients than in non-Hp-infected patients. Because aspirin or NSAIDs inhibit cyclooxygenase (COX) and prostaglandin (PG) production at inflamed sites and may increase the risk of mucosal damage in the small intestine $[18,19]$, the incidence of enteritis was determined in patients not receiving aspirin/NSAIDs; the results of this evaluation revealed that the rate of enteritis was significantly lower in the $H p$-infected patients than in the $H p$-noninfected patients. These findings suggest that $H$. pylori infection might protect the small intestinal mucosa against injury.

The possible reason for the low rate of enteritis in patients with $H$. pylori infection is that $H$. pylori can cause pangastritis and reduce gastric acid production [20-23], thereby raising the $\mathrm{pH}$ of the small intestine and protecting against small intestinal mucosal injury.

Another reason is that $H$. pylori may play an important role in the intestinal mucosal barrier function and establish a stable relationship with the intestinal bacterial flora, thereby protecting the small intestinal mucosa against damage.

There has been no evidence to date indicating that $H$. pylori may protect against small intestinal mucosal injury. Castro-Rodriguez et al. reported that $H$. pylori infection was not associated with acute or persistent diarrheal disease [24].

Recently, it has been reported that PPIs can exacerbate NSAID-induced small intestinal mucosal injury by inducing dysbiosis [6]. The PPIs, omeprazole and lansoprazole, significantly exacerbated naproxen- and celecoxib-induced intestinal ulceration and bleeding. While omeprazole did not result in mucosal injury or inflammation, it produced marked shifts in the numbers and types of enteric bacteria, including a significant reduction in the jejunal Actinobacteria and Bifidobacterium spp. Restoration of small intestinal Actinobacteria numbers through administration of selected (Bifidobacteriaenriched) commensal bacteria during treatment with omeprazole and naproxen prevented intestinal ulceration and bleeding. In other words, the enteritis induced by PPI use appeared to result from destruction of the intestinal bacterial flora.

Our study indicated that among $\mathrm{Hp}$-infected patients receiving aspirin/NSAIDs, the incidence of enteritis tended to be higher in the patient group receiving PPIs than in the patient group not receiving PPIs. The reason is that in addition to exacerbating NSAID-induced small intestinal mucosal injury, as mentioned above, PPIs also exert bacteriostatic activity against $H$. pylori $[7-9,25]$. For similar reasons, there were no significant differences in the incidence of enteritis between the patients receiving PPIs and those not receiving PPIs or between the $H p$-infected and $H p$-uninfected patient groups.

In conclusion, $H$. pylori infection was found to be associated with a reduced incidence of enteritis. Our findings suggest that $H$. pylori might protect against small intestinal mucosal injury and play an important role in the intestinal 
mucosal barrier function. Further studies are needed for a more precise elucidation of the influence of $H$. pylori on the small intestinal mucosa.

\section{References}

[1] R. Godlewska, A. Dzwonek, M. Mikuła et al., "Helicobacter pylori protein oxidation influences the colonization process," International Journal of Medical Microbiology, vol. 296, no. 4-5, pp. 321-324, 2006.

[2] R. Hirasawa, M. Tatsuta, H. Iishi et al., "Increase in apoptosis and decrease in ornithine decarboxylase activity of the gastric mucosa in patients with atrophic gastritis and gastric ulcer after successful eradication of Helicobacter pylori," American Journal of Gastroenterology, vol. 94, no. 9, pp. 2398-2402, 1999.

[3] S. Honda, T. Fujioka, M. Tokieda, T. Gotoh, A. Nishizono, and M. Nasu, "Gastric ulcer, atrophic gastritis, and intestinal metaplasia caused by Helicobacter pylori infection in Mongolian gerbils," Scandinavian Journal of Gastroenterology, vol. 33, no. 5, pp. 454-460, 1998.

[4] R. A. Veenendaal, J. M. Gotz, V. Schroijen et al., "Diagnosis of Helicobacter pylori infection by specific gastric mucosal IgA and IgG pylori antibodies," Journal of Clinical Pathology, vol. 48, no. 11, pp. 990-993, 1995.

[5] C. Granberg, A. Mansikka, O. P. Lehtonen et al., "Diagnosis of Helicobacter pylori infection by using Pyloriset EIA-G and EIAA for detection of serum immunoglobulin $\mathrm{G}$ (IgG) and IgA antibodies," Journal of Clinical Microbiology, vol. 31, no. 6, pp. 1450-1453, 1993.

[6] J. L. Wallace, S. Syer, E. Denou et al., "Proton pump inhibitors exacerbate NSAID-induced small intestinal injury by inducing dysbiosis," Gastroenterology, vol. 141, no. 4, pp. 1314-322, 2011.

[7] M. Gschwantler, B. Dragosics, H. Wurzer, G. Brandstätter, and W. Weiss, "Eradication of Helicobacter pylori by a 1-week course of famotidine, amoxicillin and clarithromycin," European Journal of Gastroenterology and Hepatology, vol. 10, no. 7, pp. 579$582,1998$.

[8] F. Mirshahi, G. Fowler, A. Patel, and G. Shaw, "Omeprazole may exert both a bacteriostatic and a bacteriocidal effect on the growth of Helicobacter pylori (NCTC 11637) in vitro by inhibiting bacterial urease activity," Journal of Clinical Pathology, vol. 51, no. 3, pp. 220-224, 1998.

[9] I. M. Nakshabendi, Q. B. Zhang, M. Mokhashi, C. G. Gemmell, F. D. Lee, and R. I. Russell, "Effect of omeprazole therapy on the survival of Helicobacter pylori, urease activity, and antral gastric histology in patients with duodenal ulcer," Helicobacter, vol. 1, no. 3, pp. 155-158, 1996.

[10] P. Malfertheiner and U. Peitz, "The interplay between Helicobacter pylori, gastro-oesophageal reflux disease, and intestinal metaplasia," Gut, vol. 54, supplement 1, pp. i13-i20, 2005.

[11] C. M. Wilcox, "Relationship between nonsteroidal anti-inflammatory drug use, Helicobacter pylori, and gastroduodenal mucosal injury," Gastroenterology, vol. 113, supplement 6, pp. S85-S92, 1997.

[12] Y. Fukuda, H. Bamba, M. Okui et al., "Helicobacter pylori infection increases mucosal permeability of the stomach and intestine," Digestion, vol. 63, supplement 1, pp. 93-96, 2001.

[13] A. Campanati, R. Gesuita, M. Giannoni et al., "Role of small intestinal bacterial overgrowth and Helicobacter pylori infection in chronic spontaneous urticaria: a prospective analysis," Acta Dermato-Venereologica, vol. 93, no. 2, pp. 161-164, 2013.
[14] E. Savarino, L. Assandri, E. G. Giannini, and V. Savarino, "Small intestinal bacterial overgrowth and Helicobacter pylori: can they be cause of thrombocytopenia in patients with chronic liver disease," American Journal of Gastroenterology, vol. 106, no. 6, pp. 1171-1172, 2011.

[15] P. Petrone, G. Sarkisyan, M. Fernández et al., "Small intestinal bacterial overgrowth in patients with lower gastrointestinal symptoms and a history of previous abdominal surgery," Archives of Surgery, vol. 146, no. 4, pp. 444-447, 2011.

[16] R. J. Dobbs, A. Charlett, S. M. Dobbs et al., "Leukocyte-subset counts in idiopathic parkinsonism provide clues to a pathogenic pathway involving small intestinal bacterial overgrowth. A surveillance study," Gut Pathogens, vol. 4, no. 1, article 12, 2012.

[17] A. Püspök, C. Dejaco, G. Oberhuber et al., "Influence of Helicobacter pylori infection on the phenotype of Crohn's disease," American Journal of Gastroenterology, vol. 94, no. 11, pp. 3239$3244,1999$.

[18] K. Higuchi, E. Umegaki, T. Watanabe et al., "Present status and strategy of NSAIDs-induced small bowel injury," Journal of Gastroenterology, vol. 44, no. 9, pp. 879-888, 2009.

[19] E. Smecuol, M. I. Pinto Sanchez, A. Suarez et al., "Low-dose aspirin affects the small bowel mucosa: results of a pilot study with a multidimensional assessment," Clinical Gastroenterology and Hepatology, vol. 7, no. 5, pp. 524-529, 2009.

[20] G. Frieri, G. De Petris, A. Aggio et al., "Gastric and duodenal juxtamucosal pH and Helicobacter pylori," Digestion, vol. 56, no. 2, pp. 107-110, 1995.

[21] A. Hamlet and L. Olbe, "The influence of Helicobacter pylori infection on postprandial duodenal acid load and duodenal bulb pH in humans," Gastroenterology, vol. 111, no. 2, pp. 391400, 1996.

[22] K. Meyer-Rosberg, D. R. Scott, D. Rex, K. Melchers, and G. Sachs, "The effect of environmental $\mathrm{pH}$ on the proton motive force of Helicobacter pylori," Gastroenterology, vol. 111, no. 4, pp. 886-900, 1996.

[23] P. Bauerfeind, R. Garner, B. E. Dunn, and H. L. T. Mobley, "Synthesis and activity of Helicobacter pylori urease and catalase at low pH," Gut, vol. 40, no. 1, pp. 25-30, 1997.

[24] J. A. Castro-Rodriguez, R. Leon-Barua, and M. Penny, "Helicobacter pylori is not a determinant factor of persistent diarrhoea or malnutrition in Peruvian children," Transactions of the Royal Society of Tropical Medicine and Hygiene, vol. 93, no. 5, pp. 537-539, 1999.

[25] S. Puiac, A. Negrea, A. Richter-Dahlfors, L. Plant, and M. Rhen, "Omeprazole antagonizes virulence and inflammation in Salmonella enterica-infected RAW264.7 cells," Antimicrobial Agents and Chemotherapy, vol. 53, no. 6, pp. 2402-2409, 2009. 


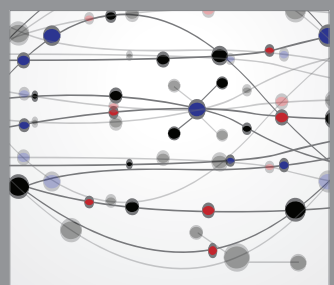

The Scientific World Journal
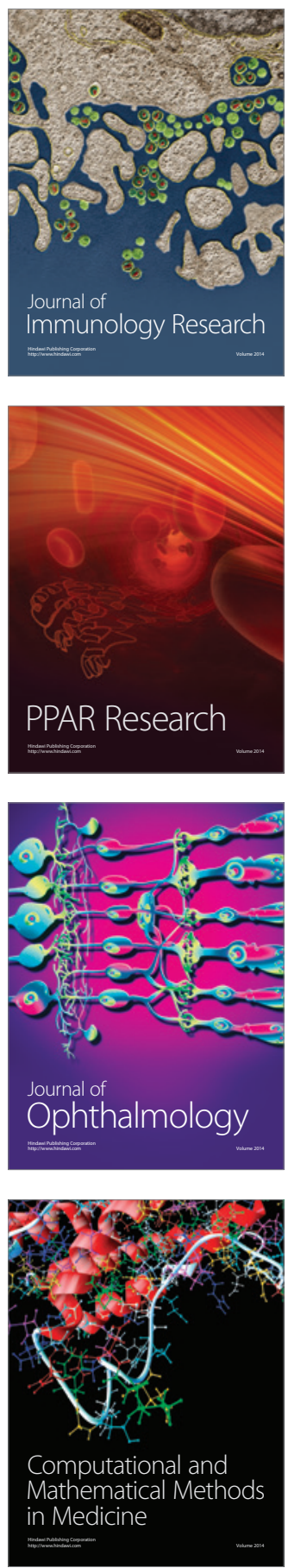

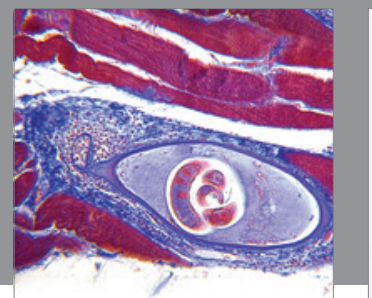

Gastroenterology

Research and Practice
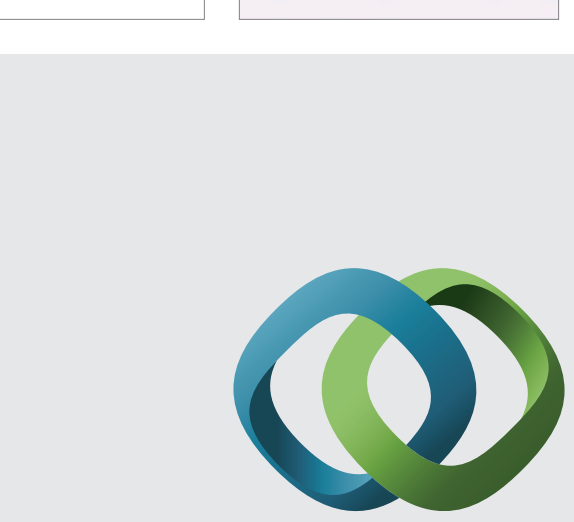

\section{Hindawi}

Submit your manuscripts at

http://www.hindawi.com
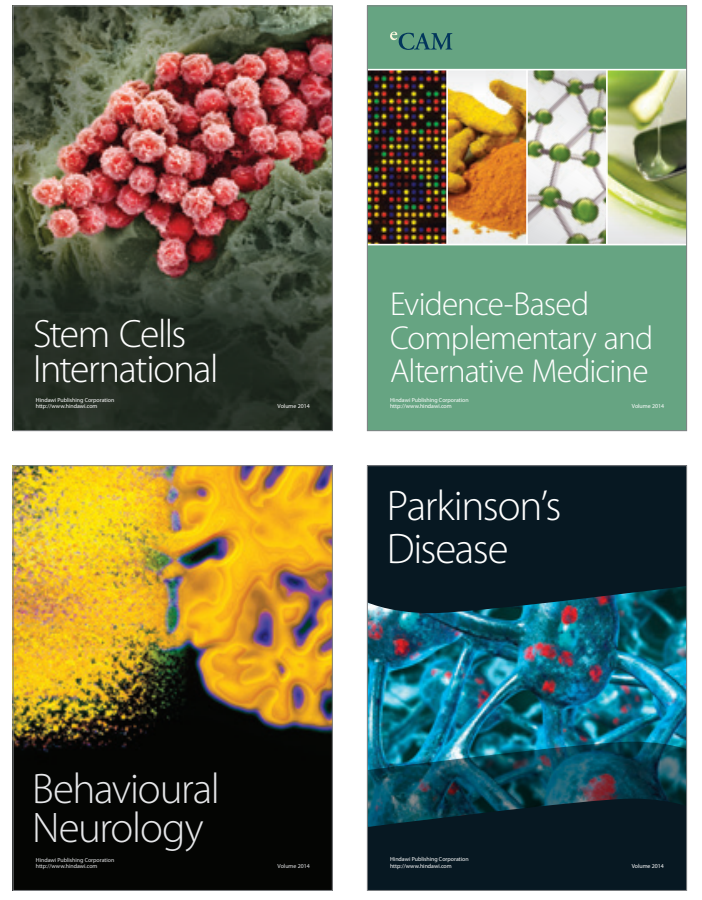
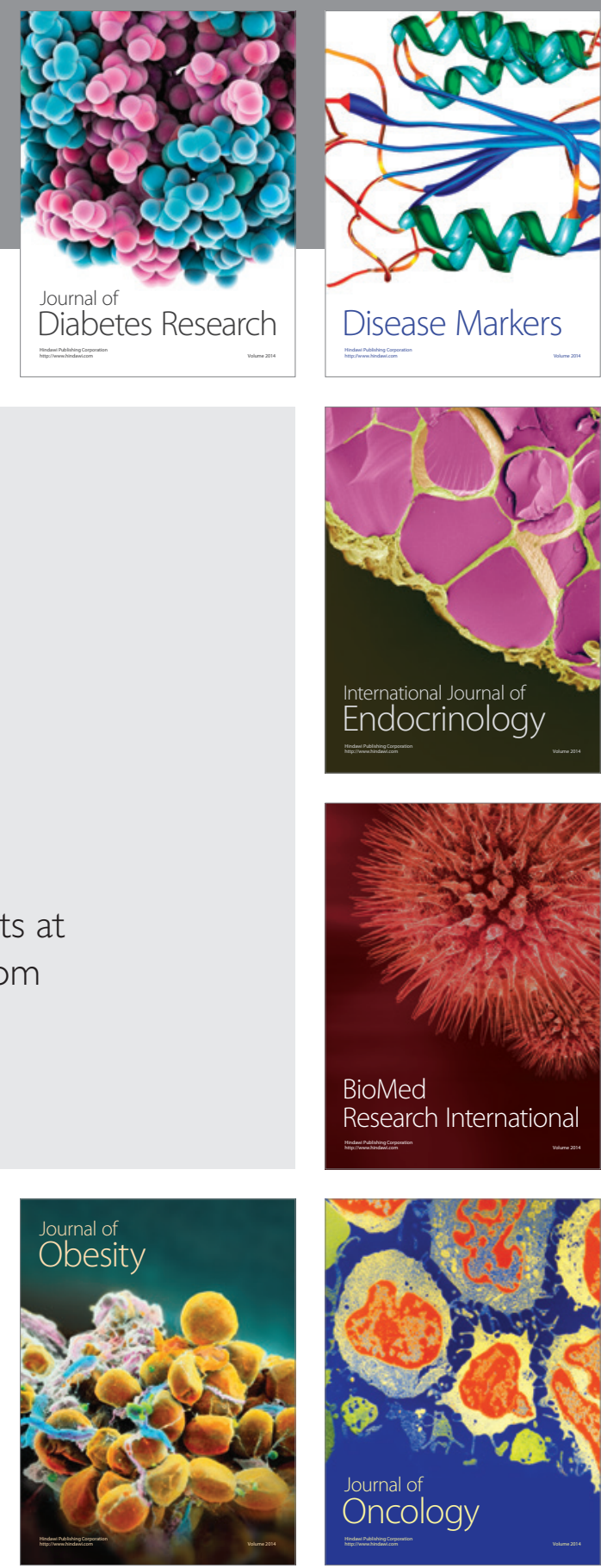

Disease Markers
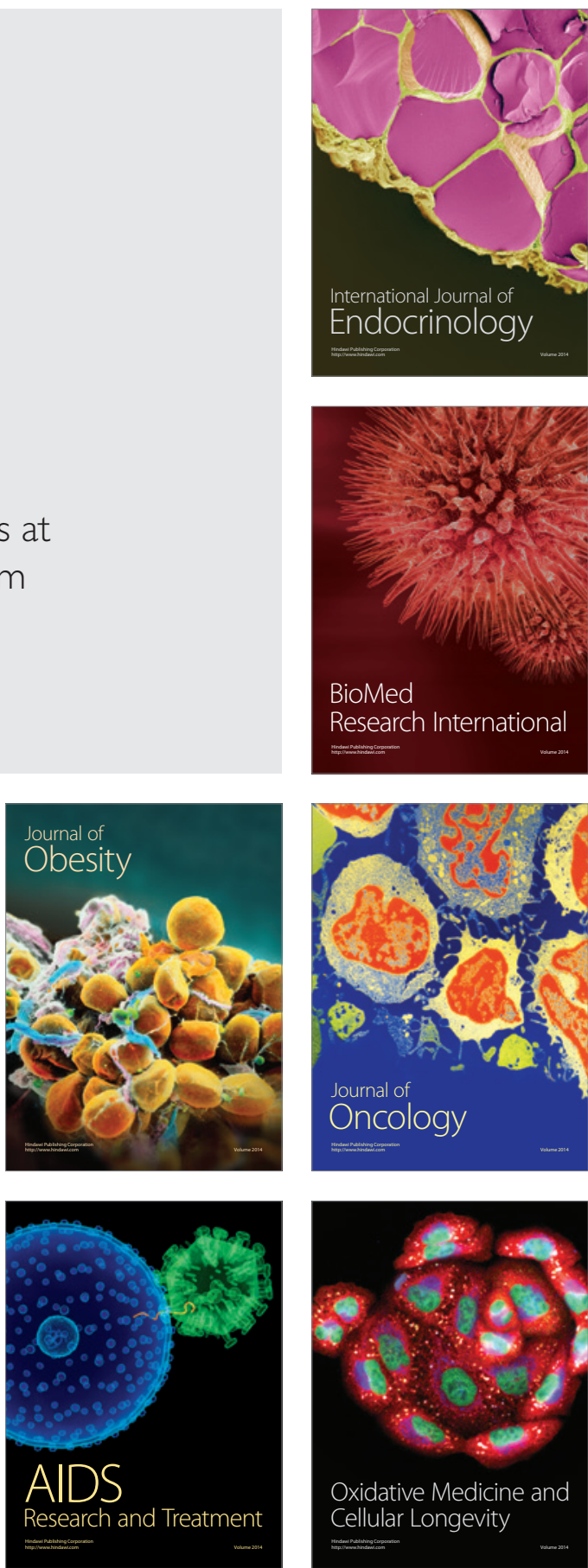